\title{
Meta-analysis of Drug Treatment for Scrub Typhus in Asia
}

\author{
Yirong Fang ${ }^{1-3}$, Zhaohui Huang ${ }^{2}$, Chunyu Tu², Lijie Zhang ${ }^{3}$, \\ Dongqing $\mathrm{Ye}^{1}$ and Bao-Ping $\mathrm{Zhu}^{3}$
}

\begin{abstract}
Objective Scrub typhus is an important febrile disease in Asia, and antibiotics have been used to treat this disease. The purpose of this study was to generate large-scale evidence of the efficacy of different antibiotic regimens for treating scrub typhus using a meta-analysis.

Methods PubMed, Elsevier ScienceDirect, Chinese Biomedical Literature Database (CBM), China National Knowledge Infrastructure (CNKI), and Wanfang (Chinese) were searched to identify relevant articles. The data from eligible citations were extracted by two reviewers. All analyses were performed using the Cochrane Collaboration Review Manager 4.2 and Stata 10.0 software programs.

Results We conducted a meta-analysis of 17 separate studies that evaluated the efficacy of treatment with the different antibiotic regimens for scrub typhus. The median time (h) to clearance of fever in the azithromycin-treated group was longer than that in the chloramphenicol-treated group (weighted mean difference $[\mathrm{WMD}]=12.66,95 \%$ confidence interval $[\mathrm{CI}]: 2.26,23.06)$. Adverse events were 2.95 (95\%CI: 1.32, 6.61) times more likely to occur in the azithromycin-treated group than in the chloramphenicol-treated group. The clearance time (days) for the main symptoms (including fever, headache, rash and lymphadenectasis) in the doxycycline-treated group was shorter than that in the chloramphenicol-treated group (WMD $=-0.4$, 95\% CI: $-0.53,-0.26$ ) in five trials. Adverse drug events occurred significantly less frequently in the azithromycin-treated group than in the doxycycline-treated group (relative risk $[\mathrm{RR}]=0.47,95 \% \mathrm{CI}$ : $0.31,0.71)$.
\end{abstract}

Conclusion Doxycycline was found to act more quickly, but more adverse drug events occur when using this regimen compared to azithromycin and chloramphenicol.

Key words: scrub typhus, randomized controlled trial, meta-analysis, treatment

(Intern Med 51: 2313-2320, 2012)

(DOI: 10.2169/internalmedicine.51.7816)

\section{Introduction}

Scrub typhus is a vector-borne zoonosis caused by Orientia tsutsugamushi, which is transmitted to humans through the bites of larval trombiculid mites $(1,2)$. People with scrub typhus develop a high fever, maculopapular rash, skin eschars, lymphadenopathy, headache, and myalgia (3). The diagnosis of scrub typhus is based on the patient's exposure history, the clinical features, and the results of serologic testing $(4,5)$. It occurs in persons who engage in occupational or recreational behavior that brings them into contact with mite-infested habitats such as brush and grass (3, 6-10). In such environments, trombiculid mite larvae can be found in any location that is suitable for rodent populations and has ground moisture sufficient to nourish the mite vectors (11).

Scrub typhus is an important cause of acute undifferentiated febrile illness in Asia (12). A tetracycline antibiotic or chloramphenicol is the conventional therapy used for the treatment of scrub typhus. Chloramphenicol and tetracycline are contraindicated in pregnant women and children, and resistance to conventional antibiotics has been reported in some papers (13). Therefore, many new antibiotics, such as quinolones and macrolides, have also been used for the treatment of scrub typhus, especially in children and pregnant women (14-16). In the past few decades, many effec-

${ }^{1}$ School of Public Health, Anhui Medical University, China, ${ }^{2}$ Department of Infectious Disease, Shaoxing Center for Disease Control and Prevention, China and ${ }^{3}$ Chinese Field Epidemiology Traning Program, Chinese Center for Disease Control and Prevention, China Received for publication March 24, 2012; Accepted for publication June 4, 2012

Correspondence to Dr. Dongqing Ye, ydq@ahmu.edu.cn 
tive antibiotics have been identified for the treatment of scrub typhus, but conflicting results have been reported for these studies.

A meta-analysis is a means of increasing the effective sample size under investigation through the pooling of data from individual association studies, thereby enhancing the statistical power (17). The previous meta-analyses have evaluated antibiotic regimens for treating scrub typhus in Korea, Thailand, Taiwan, and Malaysia, but have not included China, which is the country with the largest population in Asia (4). The aim of the present study was to perform a more complete meta-analysis to evaluate the antibiotic regimens used for the treatment of scrub typhus.

\section{Materials and Methods}

\section{Identification and selection of relevant studies}

Data were collected from the following electronic databases: PubMed, Elsevier ScienceDirect, the Cochrane Library, the Chinese Biomedical Literature Database (CBM), the China National Knowledge Infrastructure (CNKI), and Wanfang (Chinese), including reports up to June 2011.

The following keywords were used for searching: "scrub typhus", "Orientia tsutsugamushi”, "treatment", "trial”, "treating", and "therapy". The references from the retrieved papers were checked for any additional studies that might have been missed. We only included data from full published papers, not meeting or conference abstracts. No language restrictions were applied to the search.

All studies that evaluated antibiotic regimens for the treatment of scrub typhus were assessed carefully with regard to the following criteria for inclusion in the meta-analysis: (i) published up to June 2011; (ii) randomized or quasirandomized studies comparing antibiotic regimens in patients with scrub typhus; (iii) patients were diagnosed with scrub typhus, as defined by the trial authors. We excluded studies that contained overlapping data. When there were multiple publications from the same population, only the largest study was included. When a study reported the results from different subpopulations, we treated them independently.

\section{Search methods and data extraction}

Two investigators searched the electronic databases and extracted data independently, using the same standard protocols. The abstracts were reviewed independently by two investigators to determine whether they met the criteria for inclusion. References in the studies were reviewed to identify additional studies. If discrepancies occurred, a third investigator performed an additional assessment. The results of the data extraction were reviewed by a third investigator. Discrepancies were resolved by discussion with our research team. We recorded the first author's name, the year of publication, source of the publication, type of study, antibiotic regimen(s) used, total sample size, inclusion criteria, method of clinical evaluation, the number of treatment and control subjects, and the treatment outcome.

\section{Definitions}

The primary outcomes in this meta-analysis, which were related to efficacy, were defined as follows: "The clearance time (days) for the main symptoms" was defined as the interval from the day when the first dose of the study drug was administered to the day when the main clinical symptoms, including fever, headache, rash and lymphadenectasis, disappeared. "The median time (hours) to clearance of fever" was defined as the interval between the time at which the first dose of the study drug was administered and the time at which the oral temperature first returned to $37.5^{\circ} \mathrm{C}$. "Treatment failure" was defined as either persistent fever or the development of any complication after at least 48 hours of treatment. "Adverse events" were defined as symptoms or signs that developed after administration of the study drug that had not been reported prior to the first dose of the antibiotic.

\section{Meta-analysis methods}

The Cochrane Collaboration Review Manager 4.2 software program (Cochrane Collaboration, http://www.cc-ims. net/RevMan/relnotes.htm) was used to calculate the relative risk (RR), weighted mean difference (WMD), and 95\% confidence intervals (CI). Fixed and random effect models were used for the statistical analysis. The heterogeneity of outcome measures among studies was assessed by the chisquare test-based Q-statistic (18). A significant Q-statistic (p $<0.10$ ) indicated heterogeneity in the outcome measures between studies, and in this case, the random effects model was used for the meta-analysis. Otherwise, the fixed effect model was used. We also quantified the effect of heterogeneity using another measure, $\mathrm{I}^{2}=100 \% \times(\mathrm{Q}-\mathrm{df}) / \mathrm{Q}(19)$. The $\mathrm{I}^{2}$ statistic measures the degree of inconsistency in the outcome measures among studies by calculating the percentage of the total variation across studies that was due to heterogeneity rather than occurring by chance.

The pooled estimate of risk was obtained by MantelHaenszel's method in the fixed effects model and by DerSimonian-Laird's method in the random effects model $(20,21)$.

The RR and 95\%CI were used as summary statistics for the comparison of the outcome measures "treatment failure" and "adverse events"; the WMD and 95\%CI were used for the outcome measures "duration of fever" and "main symptom clearance time".

\section{Evaluation of publication bias}

Publication bias was investigated with the funnel plot, in which the standard error of $\log \mathrm{OR}$ for each study was plotted against its OR (odds ratio). Funnel plot asymmetry was assessed further using Egger's linear regression test (17). If there is asymmetry, the regression line will not run through the origin; the intercept $\alpha$ provides a measure of asymmetry. 
Table 1. Characteristics of 17 Key Treatment Studies in Scrub Typhus by June 2011

\begin{tabular}{|c|c|c|c|c|}
\hline Reference & $\begin{array}{l}\text { Type of } \\
\text { study }\end{array}$ & Number of patients & Drugs & Outcome \\
\hline Sheehy et al (14) & RCT & 60 & $\mathrm{CHL} / \mathrm{TET}$ & Duration of fever, Afebrile in 48 hours, Relapse \\
\hline Kim et al (15) & QRCT & 92 & TET vs DOX & Fever clearance time, Cure, Failure, Relapse, Toxicity and adverse events \\
\hline Brown et al (22) & RCT & 55 & DOX / TET & Afebrile in 48 hours, Disappearance of main symptoms, Side effects \\
\hline Song et al (23) & RCT & 116 & DOX / TET & Treatment failure, Relapse, Duration of fever, Disappearance of main symptoms, Side effects \\
\hline Zhong et al (24) & RCT & 62 & CHL / DOX & Cure, Failure, Fever clearance time, Hospital stays \\
\hline Quan et al (26) & RCT & 54 & QUI / CHL & Cure, Adverse drug effect, Fever clearance time \\
\hline Kim et al (27) & $\mathrm{RCT}$ & 93 & AZI / DOX & Time to defervescence, Cure. Failure, Adverse drug effect \\
\hline Wei et al (28) & RCT & 62 & $\mathrm{CHL} / \mathrm{AZI}$ & Cure, Adverse drug effect \\
\hline Li et al (29) & QRCT & 20 & $\mathrm{DOX} / \mathrm{CHL}$ & Fever clearance time, Cure, Disappearance of main symptoms, Adverse drug effect. \\
\hline Yang et al (30) & QRCT & 62 & $\mathrm{ROX} / \mathrm{CHL}$ & Cure, Disappearance of main symptoms \\
\hline Wu et al (31) & RCT & 105 & $\mathrm{CHL} / \mathrm{AZI}$ & Disappearance of main symptoms, Fever clearance time, Cure, Adverse drug effect \\
\hline Phimda et al (32) & RCT & 57 & AZI / DOX & Cure, Failure, Defervescence, Adverse events \\
\hline Chen et al (34) & QRCT & 279 & $\begin{array}{l}\text { DOX, ROX, } \\
\text { AZI, CHL, ERV }\end{array}$ & Fever clearance time, Cure. Adverse drug effect \\
\hline Chen et al (35) & QRCT & 172 & $\begin{array}{l}\text { DOX, CHL, } \\
\text { ROX }\end{array}$ & Cure, Disappearance of main symptoms \\
\hline Feng et al (36) & QRCT & 210 & $\begin{array}{l}\text { CHL, AZI, } \\
\text { LEV, DOX }\end{array}$ & Cure, Disappearance of main symptoms \\
\hline
\end{tabular}

NOTE. DOX: doxycycline, CHL: chlorampheniol, AZI: azithromycin, RIF: rifampicin, ROX: roxithromycin, TET: tetracycline, QUI: quinolones, LEV: levofloxacin, ERV: erythromycin, RCT: Randomized controlled trial, QRCT: Quasi-randomized controlled trial

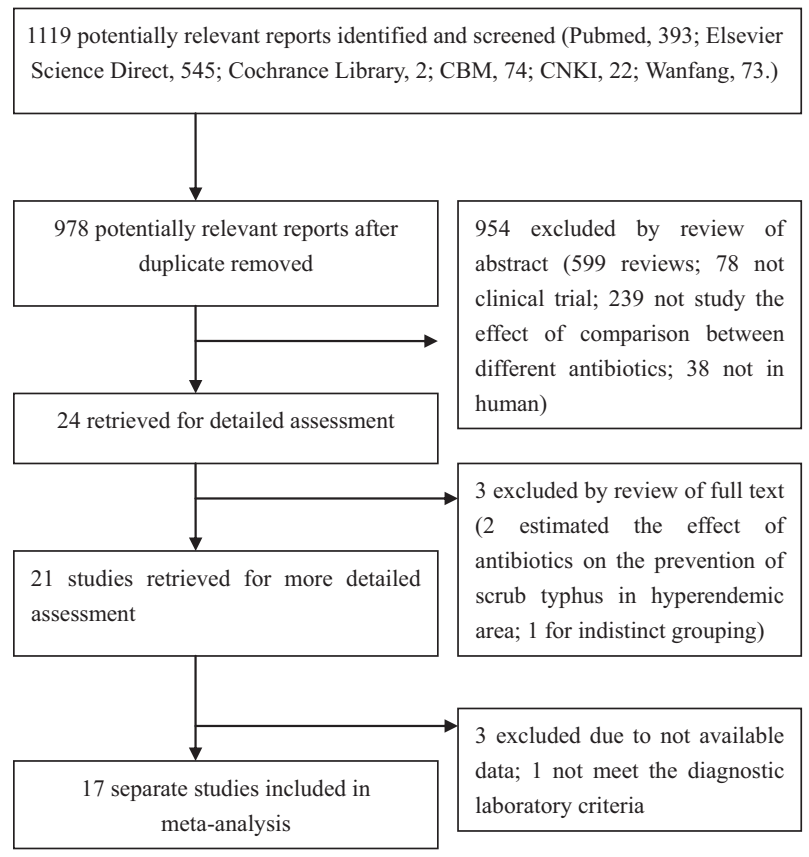

Figure 1. Flow diagram for selecting a study for meta-analysis of drug treatment for scrub typhus.

The larger its deviation from zero, the more pronounced the asymmetry. The analyses were performed using the Stata version 10 software program (StataCorp LP, College Station, TX, USA). All $\mathrm{p}$ values were two-sided. A $\mathrm{p}$ value $<0.05$ was considered to be statistically significant.

Results

\section{Characteristics of eligible studies}

The characteristics of studies comparing the efficacy of antibiotics in the treatment of scrub typhus are presented in Table 1 . There were 1,119 papers potentially relevant to the search terms (PubMed: 393; Elsevier ScienceDirect: 545; Cochrane Library: 2; CBM: 74; CNKI: 22; Wanfang: 73). The study selection process is shown in Fig. 1. There were 978 potentially relevant studies after duplicates were removed. During the step of screening the abstracts, 954 of these articles were excluded (599 were review articles; 78 were not clinical trials; 239 did not compare different antibiotics; 38 studies did not involve humans). This left 24 studies for full publication review; of these, 3 were excluded (2 estimated the effect of antibiotics on the prevention of scrub typhus in a hyper-endemic area; 1 had indistinct grouping). The remaining 21 studies were retrieved for more detailed evaluation; 3 were excluded due to unavailable data, 1 did not meet the diagnostic laboratory criteria and was excluded.

There were therefore 17 studies in the meta-analysis: 9 of them were carried out in China, 3 in Korea, 2 in Thailand, 2 in Taiwan, and 1 in Malaysia (Table 1). The meta-analysis was performed for six antibiotics (doxycycline, chloramphenicol, azithromycin, rifampicin, roxithromycin, and tetracycline). The data comparing tetracycline versus chloramphenicol was extracted only from the study by Sheehy (14), and the contrast of teilithromycin versus doxycycline was only extracted from the study by Kim (15), so a metaanalysis was not performed for these comparisons. 
Table 2. Meta-analysis of Drug Treatment for Scrub Typhus in 17 Separate Studies

\begin{tabular}{|c|c|c|c|c|c|c|c|c|c|c|c|}
\hline \multirow[b]{2}{*}{ Outcome } & \multirow[b]{2}{*}{ Comparisons } & \multicolumn{2}{|c|}{ Sample size } & \multirow{2}{*}{$\begin{array}{c}\text { No. } \\
\text { of } \\
\text { studies }\end{array}$} & \multicolumn{3}{|c|}{ Test of drug treatment } & \multicolumn{4}{|c|}{ Test of heterogeneity } \\
\hline & & $\mathrm{T}$ & $\mathrm{C}$ & & RR/WMD(95\%CI) & $Z$ & $\mathrm{p}$ & Model & $\chi^{2}$ & $\mathrm{p}$ & $I^{2} \%$ \\
\hline \multirow[t]{4}{*}{ AZI VS CHL(Ref) } & Duration of fever & 128 & 174 & 4 & $12.66(2.26,23.06)$ & 2.39 & 0.02 & $\mathrm{R}$ & 81.86 & $<0.01$ & 96.3 \\
\hline & Symptom clearance time & 159 & 127 & 4 & $-0.22(-1.83,1.38)$ & 0.27 & 0.78 & $\mathrm{R}$ & 254.58 & $<0.01$ & 98.8 \\
\hline & Treatment failure & 201 & 217 & 5 & $1.58(0.69,3.65)$ & 1.08 & 0.28 & $\mathrm{~F}$ & 0.80 & 0.67 & 0 \\
\hline & Adverse events & 123 & 120 & 4 & $2.95(1.32,6.61)$ & 2.64 & $<0.01$ & $\mathrm{~F}$ & 0.12 & 0.99 & 0 \\
\hline \multirow[t]{3}{*}{ DOX VS CHL(Ref) } & Duration of fever & 157 & 148 & 5 & $-3.98(-8.17,0.21)$ & 1.86 & 0.06 & $\mathrm{R}$ & 19.34 & $<0.01$ & 84.5 \\
\hline & Symptom clearance time & 172 & 166 & 5 & $-0.40(-0.53,-0.26)$ & 5.77 & $<0.01$ & $\mathrm{~F}$ & 9.01 & 0.06 & 55.6 \\
\hline & Treatment failure & 255 & 252 & 6 & $0.83(0.42,1.65)$ & 0.53 & 0.60 & $\mathrm{~F}$ & 2.05 & 0.56 & 0 \\
\hline \multirow[t]{4}{*}{ AZI VS DOX(Ref) } & Duration of fever & 76 & 122 & 3 & $9.54(-2.71,21.19)$ & 1.53 & 0.13 & $\mathrm{R}$ & 80.08 & $<0.01$ & 97.5 \\
\hline & Symptom clearance time & 107 & 104 & 3 & $-0.33(-1.80,1.14)$ & 0.44 & 0.66 & $\mathrm{R}$ & 40.65 & $<0.01$ & 95.1 \\
\hline & Treatment failure & 195 & 225 & 5 & $0.98(0.43,2.24)$ & 0.04 & 0.97 & $\mathrm{~F}$ & 3.12 & 0.21 & 35.9 \\
\hline & Adverse events & 226 & 221 & 3 & $0.47(0.31,0.71)$ & 3.57 & $<0.01$ & $\mathrm{~F}$ & 2.14 & 0.34 & 6.3 \\
\hline \multirow[t]{2}{*}{ ROX VS DOX(Ref) } & Symptom clearance time & 74 & 68 & 2 & $1.04(-2.09,4.16)$ & 0.65 & 0.51 & $\mathrm{R}$ & 42.14 & $<0.01$ & 97.6 \\
\hline & Treatment failure & 125 & 146 & 3 & $1.30(0.55,3.07)$ & 0.59 & 0.55 & $\mathrm{~F}$ & 0.15 & 0.70 & 0 \\
\hline RIF VS DOX(Ref) & Adverse events & 74 & 58 & 2 & $0.70(0.43,1.14)$ & 1.42 & 0.15 & $\mathrm{~F}$ & 0.04 & 0.84 & 0 \\
\hline TET VS DOX(Ref) & Adverse events & 74 & 91 & 2 & $0.54(0.01,25.04)$ & 0.31 & 0.75 & $\mathrm{R}$ & 7.29 & $<0.01$ & 86.3 \\
\hline
\end{tabular}

NOTE. The RR and $95 \%$ CI were used as summary statistics for the comparison of outcome measures of "treatment failure (\%)" and "adverse events (\%)", the WMD and $95 \% \mathrm{CI}$ were used for the outcome measures of "duration of fever (hours)" and "main symptom clearance time(days)".

T: Treatment, C: Control, DOX: doxycycline, CHL: chlorampheniol, AZI: azithromycin, RIF: rifampicin, ROX: roxithromycin, TET: tetracycline, VS: vesvus, R: random effect model, F: fixed effect model, RR: relative risk, WMD :weighted mean differences, CI 95\%: 95\% confidence intervals, Ref: reference

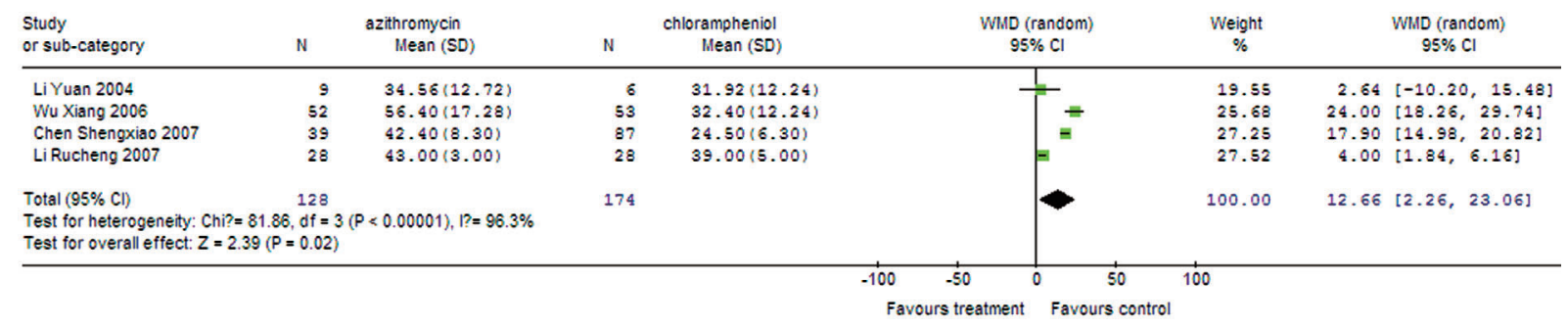

Figure 2. Forest plots for meta-analysis of duration of fever (hours) between azithromycin and chlorampheniol.

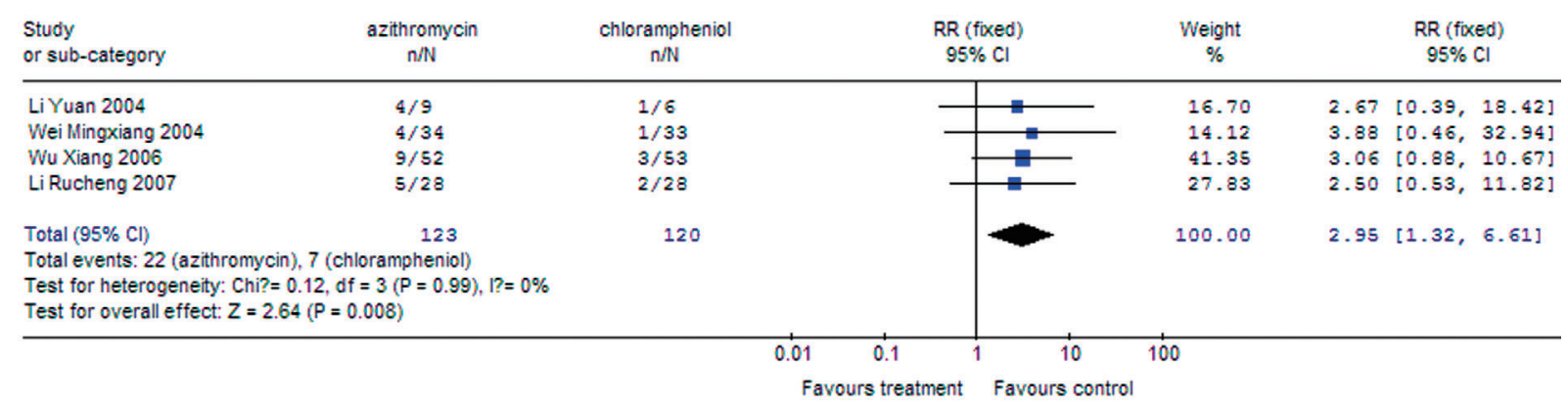

Figure 3. Forest plots for meta-analysis of adverse events (\%) between azithromycin and chlorampheniol.

\section{Meta-analysis of the effects of azithromycin and chloramphenicol}

Five separate trials compared azithromycin with chloramphenicol, and the main results are presented in Table 2. The Q-test of heterogeneity was not significant for treatment failure and adverse events, and we conducted the analyses using fixed effects models; however, the Q-test of heterogeneity was significant for the duration of fever and symptom clearance time, so we conducted the analyses using random effects models. The median fever clearance time (h) in the azithromycin-treated group was longer than that in the chloramphenicol-treated group (weighted mean difference [WMD] $=12.66$, CI95\%: 2.26, 23.06). Adverse events were 2.95 (CI95\%: 1.32, 6.61) times more likely to occur in the azithromycin group than in the chlorampheniol group. The forest plots of positive results are shown in Fig. 2 and 3. We could not detect any difference in either the symptom clear- 


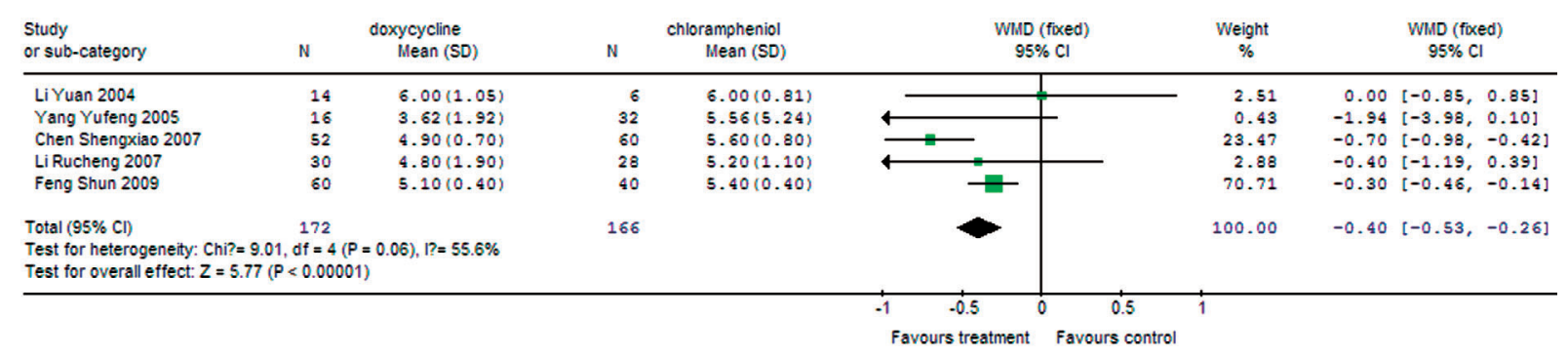

Figure 4. Forest plots for meta-analysis of main symptom clearance time (days) between doxycycline and chlorampheniol.

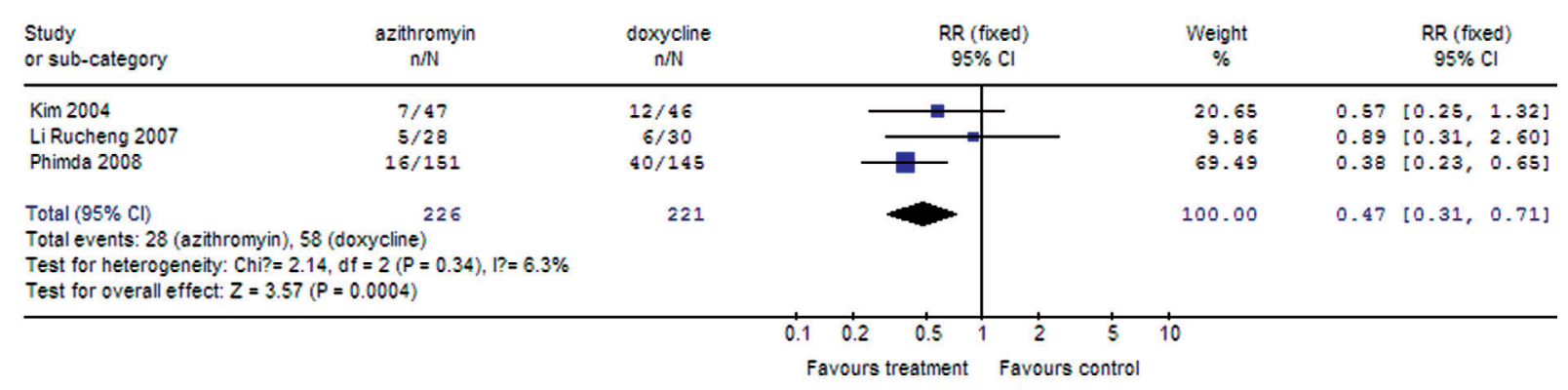

Figure 5. Forest plots for meta-analysis of adverse events (\%) between azithromycin and doxycycline.

ance time or treatment failure.

\section{Meta-analysis of the effects of doxycycline and chlo- ramphenicol}

Six separate trials compared doxycycline with chloramphenicol. The main symptom clearance time (days) (including fever, headache, rash, and lymphadenectasis) in the doxycycline-treated group was shorter than that in the chloramphenicol group (WMD $=-0.4$, CI95\%: $-0.53,-0.26)$ (Table 2), and the forest plot of the distribution of the RRs is shown in Fig. 4.

\section{Meta-analysis of the effects of azithromycin and doxycycline}

Three trials compared azithromycin with doxycycline. No difference was detected between azithromycin and doxycycline in the duration of fever, symptom clearance time, and treatment failure. However, adverse events were less likely to occur in the azithromycin-treated group than in the doxycycline-treated group ( $R R=0.47, \mathrm{CI} 95 \%$ : $0.31,0.71)$ (Table 2), and the forest plot of the distribution of the RRs is shown in Fig. 5.

\section{Meta-analysis of the effects of roxithromycin and doxycycline}

The differences between the effects of roxithromycin and doxycycline were investigated in three separate trials. We found no significant differences between roxithromycin and doxycycline in terms of treatment failure and the symptom clearance time (Table 2).

\section{Meta-analysis of the effects of rifampicin and doxy- cycline}

Two trials compared rifampicin and doxycycline. We found no significant difference between rifampicin and doxycycline in the occurrence of adverse events (Table 2).

\section{Meta-analysis of the effects of tetracycline and doxy- cycline}

Two trials compared tetracycline and doxycycline. We found no significant differences between tetracycline and doxycycline in the incidence of adverse events (Table 2).

\section{Evaluation of publication bias}

We assessed the funnel plot asymmetry using Egger's linear regression test. The results are shown in Table 3. There was no publication bias in most comparisons. However, Egger's test was not applied in two comparisons because of the small number of studies (rifampicin versus doxycycline and tetracycline versus doxycycline).

\section{Conclusion}

We herein assessed the effects of the treatment of scrub typhus with antibiotics. Ten randomized controlled trials and seven quasi-randomized controlled trials were included in our meta-analyses. We found that chloramphenicol exerted a more rapid therapeutic effect compared to azithromycin. Doxycycline acted even more quickly than chloramphenicol, but it was also associated with more adverse drug events compared to azithromycin. 
Table 3. Egger's Linear Regression Test of Funnel Plot Asymmetry

\begin{tabular}{|c|c|c|c|c|}
\hline \multirow[b]{2}{*}{ Drug } & \multicolumn{4}{|c|}{ Y-axis intercept: a $(95 \% \mathrm{CI})$} \\
\hline & Duration of fever & $\begin{array}{c}\text { Symptom clearance } \\
\text { time }\end{array}$ & Treatment failure & Adverse events \\
\hline $\begin{array}{l}\text { DOX VS } \\
\text { CHL }\end{array}$ & $-0.39(-15.65,14.85)$ & $3.49(-0.82,7.79)$ & $0.41(-8.35,-9.17)$ & $0.44(-6.99,7.87)$ \\
\hline $\begin{array}{l}\text { AZI VS } \\
\text { CHL }\end{array}$ & $-5.79(-29.10,17.53)$ & $-11.86(-63.36,39.65)$ & $-1.51(-18.14,15.12)$ & NA \\
\hline $\begin{array}{l}\text { AZI VS } \\
\text { DOX }\end{array}$ & $-16.52(-180.63,147.60)$ & $1.28(-432.11,434.67)$ & NA & $0.10(-3.49,3.69)$ \\
\hline $\begin{array}{l}\text { ROX VS } \\
\text { DOX }\end{array}$ & NV & NA & NA & NA \\
\hline $\begin{array}{l}\text { RIF VS } \\
\text { DOX }\end{array}$ & NA & NA & NA & NA \\
\hline $\begin{array}{l}\text { TET VS } \\
\text { DOX }\end{array}$ & NA & NA & NA & NA \\
\hline
\end{tabular}

One of these trials, conducted by Sheehy in 1973 (14), was based on the use of clinical features to diagnose scrub typhus, and this may have resulted in a selection bias. Twelve of the trials, in which the diagnosis of scrub typhus was made based on clinical manifestations and a 4-fold rise in the Weil-Felix (WF) titer to at least 1:160, included patients who had been in contact with mite-infested habitats such as brush and grass. Patients diagnosed with scrub typhus by more strict serologic or molecular methods were enrolled in four trials $(15,25,27,32)$. Strict and specific case ascertainment increases the research validity of a metaanalysis.

A meta-analysis of seven trials performed by Panpanich (4) evaluated the antibiotic regimens used for the treatment of scrub typhus in Korea, Thailand, Taiwan, and Malaysia, but did not include China, which has the largest population in Asia. Therefore, we included nine trials conducted in China in our meta-analysis, and selected four treatment outcomes: duration of fever, clearance time for the main symptoms, treatment failure, and adverse events, to more comprehensively reflect the antibiotic efficacy. The lack of differences detected in the study by Panpanich may have been due to the relatively limited trials and small populations used in their analysis (4). Therefore, more studies based on larger sample size and a randomized controlled trial design are needed in future research.

One of the earliest antibiotics to be used in the treatment of scrub typhus was chloramphenicol. However, an early study showed that tetracycline was more effective; in view of the risk of aplastic anemia, chloramphenicol is now seldom used to treat scrub typhus, and is best avoided in pregnant women and children (37). Tetracyclines are used widely for the treatment of scrub typhus, but they are also contraindicated during pregnancy because of their teratogenicity and should not be given to children because they may cause permanent staining of the teeth (38). Both the meta-analysis by Panpanich (4) and the review by Raja- pakse (37) confirmed the efficacy of doxycycline. In our study, we retrieved six studies that compared doxycycline and chlorampheniol; our meta-analysis showed that doxycycline was superior to chlorampheniol in the relief of symptoms. Treatment with doxycycline is associated with a rapid abatement of clinical manifestations, and oral doxycycline is the currently standard treatment for mild cases of scrub typhus (32). A study conducted by Tsai reported the response rate and the time to defervescence in patients with uncomplicated scrub typhus treated with intravenous minocycline to not be different from those of patients treated with oral doxycycline. Oral doxycycline is also more convenient to use and much cheaper than intravenous minocycline for patients with noncomplicated scrub typhus (39).

Macrolides such as azithromycin have been reported to be equally effective as doxycycline for the treatment of scrub typhus, and gastrointestinal manifestations are significantly less frequent with azithromycin. Azithromycin is more expensive than docycycline, but it is easier to administer and is also better tolerated $(4,38)$, the latter result was confirmed in the current meta-analysis. In this study, we retrieved five studies that included 195 cases treated with azithromycin and 225 cases treated with doxycycline. In the evaluation of treatment failure, we found no difference between these two antibiotics. Roxithromycin is one of the new generation macrolide antibiotics, but in our investigation, no differences in either treatment failure or the main symptom clearance time were detected between roxithromycin and doxycycline. In a trial conducted in China by Chen and coworkers, doxycycline was shown to be superior to roxithromycin in terms of its faster defervescence (34).

A randomized controlled trial comparing rifampicin and doxycycline reported that fewer patients in the rifampicintreated group remained febrile at 48 hours (25), the clearance time for fever in the rifampicin group was about half of that in the doxycycline-treated group, and mild gastrointestinal symptoms were common in both groups. In our 
meta-analysis, which included two trials, we did not detect any differences in adverse events between rifampicin and doxycycline.

Several specific details of this meta-analysis merit consideration. First, only published studies were included, and some publication bias may thus have occurred, even though the use of a statistical test did not show it. Second, a metaanalysis is a form of retrospective research that is subject to the methodological deficiencies of the studies included in the analysis. Third, the concealment of allocation was poorly reported, and only one study included in this review reported adequate allocation concealment (32). Finally, the diagnostic criteria for scrub typhus varied widely in these clinical trials, and it is possible that some cases of suspected scrub typhus were due to other infections that give rise to similar symptoms and clinical findings.

In summary, the present meta-analysis indicated that doxycycline can take effect more quickly, but with more adverse drug events, compared to azithromycin and chloramphenicol. Macrolide antibiotics such as azithromycin were found to be highly effective against scrub typhus. They are appropriate alternatives in areas where doxycycline-resistant scrub typhus is prevalent, and also for children under 8 years of age or women during pregnancy, in whom doxycycline is contraindicated (32).

The authors state that they have no Conflict of Interest (COI).

\section{References}

1. Watt G, Parola P. Scrub typhus and tropical rickettsioses. Curr Opin Infect Dis 16: 429-436, 2003.

2. Ogawa M, Hagiwara T, Kishimoto T, et al. Scrub typhus in Japan: epidemiology and clinical features of cases reported in 1998. Am J Trop Med Hyg 67: 162-165, 2002.

3. Razak A, Sathyanarayanan V, Prabhu M, Sangar M, Balasubramanian R. Scrub typhus in Southern India: are we doing enough? Trop Doct 40: 149-151, 2010.

4. Panpanich R, Garner P. Antibiotics for treating scrub typhus. Cochrane Database Syst Rev 7: CD002150, 2010.

5. Lee CS, Min IS, Hwang JH, Kwon KS, Lee HB. Clinical significance of hypoalbuminemia in outcome of patients with scrub typhus. BMC Infect Dis 10: 216, 2010.

6. Sharma PK, Ramakrishnan R, Hutin YJ, et al. Scrub typhus in Darjeeling, India: opportunities for simple, practical prevention measures. Trans R Soc Trop Med Hyg 103: 1153-1158, 2009.

7. Watt G, Chouriyagune C, Ruangweerayud R, et al. Scrub typhus infections poorly responsive to antibiotics in northern Thailand. Lancet 348: 86-89, 1996.

8. Graves S, Stenos J. Rickettsioses in Australia. Ann NY Acad Sci 1166: 151-155, 2009.

9. Hashimoto S, Kawado M, Murakami Y, et al. Epidemics of vectorborne diseases observed in infectious disease surveillance in Japan, 2000-2005. J Epidemiol 17 (Suppl): S48-S55, 2007.

10. Liu YX, Jia N, Suo JJ, Xing YB, Liu G, Xiao HJ. Characteristics of pediatric scrub typhus in a new endemic region of northern China. Pediatr Infect Dis J 28: 1111-1114, 2007.

11. Kawamura A, Tanaka H, Tamura A. Tsutsugamushi disease. University of Tokyo Press, Tokyo, 1995.

12. Chrispal A, Boorugu H, Gopinath KG, et al. Scrub typhus: an un- recognized threat in South India: clinical profile and predictors of mortality. Trop Doct 40: 129-133, 2010.

13. Watt G, Chouriyagune C, Ruangweerayud R, et al. Scrub typhus infections poorly responsive to antibiotics in northern Thailand. Lancet 348: 86-89, 1996.

14. Sheehy TW, Hazlett D, Turk RE. Scrub typhus. A comparison of chloramphenicol and tetracycline in its treatment. Arch Intern Med 132: 77-80, 1973.

15. Kim DM, Yu KD, Lee JH, Kim HK, Lee SH. Controlled trial of a 5-day course of telithromycin versus doxycycline for treatment of mild to moderate scrub typhus. Antimicrob Agents Chemother 51: 2011-2015, 2007.

16. Tsai CC, Lay CJ, Wang CL, Ho YH, Wang LS, Chen LK. Levofloxacin versus tetracycline antibiotics for the treatment of scrub typhus. Int J Infect Dis 14: 62-67, 2010.

17. Egger M, Davey SG, Schneider M, Minder C. Bias in metaanalysis detected by a simple, graphical test. BMJ 315: 629-634, 1997.

18. Cochran WG. The combination of estimates from different experiments. Biometrics 1954: 101-129, 2010.

19. Higgins JP, Thompson SG. Quantifying heterogeneity in a metaanalysis. Stat Med 21: 1539, 2002.

20. DerSimonian R, Laird N. Meta-analysis in clinical trials. Control Clin Trials 7: 177-188, 1986.

21. Mantel N, Haenszel W. Statistical aspects of the analysis of data from retrospective studies of disease. J Natl Cancer Inst 22: 719$748,1959$.

22. Brown GW, Saunders JP, Singh S, Huxsoll DL, Shirai A. Single dose doxycycline therapy for scrub typhus. Trans R Soc Trop Med Hyg 72: 412-416, 1978.

23. Song JH, Lee C, Chang WH, et al. Short-course doxycycline treatment versus conventional tetracycline therapy for scrub typhus: a multicenter randomized trial. Clin Infect Dis 21: 506-510, 1995.

24. Zhong ZY, Zhang WY, Wu YX. Scrub typhus. A comparison of doxycycline and chloramphenicol in its treatment. J Guangdong Med Coll 14: 354-355, 1996 (in Chinese).

25. Watt G, Kantipong P, Jongsakul K, Watcharapichat P, Phulsuksombati D, Strickman D. Doxycycline and rifampicin for mild scrub-typhus infections in northern Thailand: a randomised trial. Lancet 356: 1057-1061, 2000.

26. Quan B. The third generation of quinolones therapy for scrub typhus. J Trop Med 2: 163-164, 2002 (in Chinese).

27. Kim YS, Yun HJ, Shim SK, Koo SH, Kim SY, Kim S. A comparative trial of a single dose of azithromycin versus doxycycline for the treatment of mild scrub typhus. Clin Infect Dis 39: 13291335, 2004.

28. Wei MX, Lin SJ, Yan R, Guo WZ. A comparison of azithromycin versus chloramphenicol for treatment of paediatric scrub typhus. J Appl Clin Pediatr 19: 66, 2004 (in Chinese).

29. Li Y. Macrolide antibiotic to cure child scrub typhus. J Pediatr Pharm 10: 36, 2004 (in Chinese).

30. Yang YF, Li B, Liu XM, Zhang LP. Effect of roxithromycin in the treatment of scrub typhus. J Pediatr Pharm 11: 32, 2005 (in Chinese).

31. Wu X, Xiao H, Zhu DJ. Therapeutic effect of azithromycin on children with tsutsugamushi disease. J Chin Pediatr 24: 769-770, 2006 (in Chinese).

32. Phimda K, Hoontrakul S, Suttinont C, et al. Doxycycline versus azithromycin for treatment of leptospirosis and scrub typhus. Antimicrob Agents Chemother 51: 3259-3263, 2007.

33. Li RC, Pang L, Lu XH. Effect of antibiotics in the treatment of scrub typhus. Mod Med Health 23: 1936-1937, 2007 (in Chinese).

34. Chen SX, Lin XM, Shen HF, Zhang LJ, Sun ZM. Treatment of scrub typhus with macrolide antibiotic. J Trop Med 7: 783-784, 2007 (in Chinese). 
35. Chen SX, Shen HF, Zhang LJ, Xu CX, Sun ZM. Effect of antibiotics in the treatment of scrub typhus in South China Sea area. J Pre Med Chin PLA 26: 59, 2008 (in Chinese).

36. Feng X. Treatment of scrub typhus with several antibiotics. J Pract Med 25: 526, 2009 (in Chinese).

37. Rajapakse S, Rodrigo C, Fernando SD. Drug treatment of scrub typhus. Trop Doct 41: 1-4, 2011.
38. Kim YS, Lee HJ, Chang M, Son SK, Rhee YE, Shim SK. Scrub typhus during pregnancy and its treatment: a case series and review of the literature. Am J Trop Med Hyg 75: 955-959, 2006.

39. Tsai CC, Lay CJ, Ho YH, Wang LS, Chen LK. Intravenous minocycline versus oral doxycycline for the treatment of noncomplicated scrub typhus. J Microbiol Immunol Infect 44: 33-38, 2011.

(C) 2012 The Japanese Society of Internal Medicine http://www.naika.or.jp/imonline/index.html 